\title{
Primary Percutaneous Coronary Intervention in a COVID-19 Patient with ST-segment Elevation Myocardial Infarction after Lung Transplantation: A Case Report
}

\section{Tian-ming XUAN}

the First Affiliated Hospital, Zhejiang University School of Medicine, Hangzhou 310003, China.

\section{Xing-xiang WANG}

Xiang-yuan PU

Xiao-gang GUO ( $\sim$ gxg22222@zju.edu.cn )

the First Affiliated Hospital, Zhejiang University School of Medicine, Hangzhou 310003, China.

Wei-li HAN

\section{Case Report}

Keywords: COVID-19, Coronavirus, Acute Respiratory Distress Syndrome, ST Elevation Myocardial Infarction, Percutaneous Coronary Revascularization, Lung Transplantation

Posted Date: April 9th, 2020

DOI: https://doi.org/10.21203/rs.3.rs-22040/v1

License: (c) (i) This work is licensed under a Creative Commons Attribution 4.0 International License.

Read Full License

Version of Record: A version of this preprint was published at Journal of Zhejiang University-SCIENCE B on May 1st, 2020. See the published version at https://doi.org/10.1631/jzus.B2000182. 


\section{Abstract}

We present an unusual case of a patient with bilateral lung transplantation due to severe Coronavirus Disease 2019 (COVID-19), who subsequently complicated with acute myocardial infarction and underwent primary percutaneous coronary intervention.

\section{Case Report}

The patient was a 70-year-old man with a history of hypertension and diabetes, which was not well controlled. He had a travel history to Hubei province at the end of January 2020. After returning to Zhejiang province on February 2, he developed fever with a body temperature of $37.8^{\circ} \mathrm{C}$, accompanied by cough and anorexia. He went to a local hospital and the nucleic acid test for novel coronavirus (2019$\mathrm{nCoV}$ ) was positive. He was diagnosed with COVID-19 and the initial treatment regimen included methylprednisolone, umifenovir and darunavir. During the treatment, the fever and cough did not improve significantly, and he progressively developed dyspnea.

On February 9, the patient was transferred to the intensive care unit of our hospital. On arrival, the patient's heart rate was 78 beats/min, respiratory rate 27 breaths/min, and blood pressure $124 / 74 \mathrm{mmHg}$. Key laboratory examinations were as follows: white blood cell count, $6.6 \times 10^{\wedge} 9 / \mathrm{L}$; neutrophil, $89.1 \%$; hemoglobin, $129 \mathrm{~g} / \mathrm{L}$; platelet count, $180 \times 10^{\wedge} 9 / \mathrm{L}$; creatinine, $64 \mu \mathrm{mol} / \mathrm{L}$; high sensitive $\mathrm{C}$ reaction protein (hs-CRP), $45.51 \mathrm{mg} / \mathrm{L}$. Computed tomography (CT) scan showed diffuse infiltrations in both lungs. After admission, he was treated with high-flow oxygen inhalation, methylprednisolone, umifenovir, piperacillin, and tazobactam. On February 14, he was intubated due to severe hypoxia. Since February 22, repeated nucleic acid tests for 2019-nCoV in sputum and broncho-alveolar lavage fluid were all negative. However, his condition continued to worsen due to pulmonary consolidation, and sputum cultures suggested Stenotrophomonas maltophilia infection. Veno-venous extracorporeal membrane oxygenation (VVECMO) treatment was started on February 26, and CT scan on March 2 revealed significant progression of pulmonary consolidation (Figure 1). After expert panel discussion, the patient received bilateral-lung transplantation on March 8. After operation, the oxygen saturation improved significantly, and the patient's condition gradually stabilized. ECMO was withdrawn and continuous renal replacement therapy (CRRT) was started on March 10. On March 11, laboratory examinations were as follows: hemoglobin, $80 \mathrm{~g} / \mathrm{L}$; platelet count, $36 \times 10^{\wedge} 9 / \mathrm{L}$; creatinine, $103 \mu \mathrm{mol} / \mathrm{L}$; international normalized ratio (INR), 1.77 ; activated partial thromboplastin time (APTT), 45.9 seconds; prothrombin time (PT), 20.8 seconds; Ddimer, $2927.00 \mu \mathrm{g} / \mathrm{L}$. On March 12, the patient developed a sudden episode of ventricular fibrillation. After cardiopulmonary resuscitation and defibrillation, sinus rhythm was restored. However, the high sensitivity cardiac troponin I (hs-cTnl) gradually increased to $23.31 \mathrm{ng} / \mathrm{mL}$. Electrocardiogram (ECG) showed dynamic change of ST segment elevation through V1 to V6 leads (Figure 2). Echocardiography suggested mildly reduced left ventricular (LV) systolic function and segmental hypokinesia of the ventricular septum and anterior LV wall. ST-segment elevation myocardial infarction (STEMI) was diagnosed and primary percutaneous coronary intervention (PCl) was performed on March 13. Coronary angiography showed severe stenosis and thrombus formation in the middle segment of left anterior 
descending (LAD) artery with TIMI grade 3 flow, and mild to moderate stenosis in the remaining vessels. A drug-eluting stent (Partner $3.0 \times 24 \mathrm{~mm}$ ) was deployed at the LAD lesion, followed by post-dilation with a non-compliant balloon $(3.25 \times 15 \mathrm{~mm}$ ). Post-procedural TIMI flow was grade 3 (Figure 2), and the patient's hemodynamics were stable. Dual antiplatelet therapy with aspirin $100 \mathrm{mg}$ and clopidogrel $75 \mathrm{mg}$ daily was administrated after intervention. ECG showed recovery of ST segment, and cTnl gradually declined. Aspirin was discontinued on the fourth day after PCI due to multiple bleeding risks.

\section{Discussion}

Since December 2019, a sudden outbreak of Coronavirus Disease 2019 (COVID-19) epidemic has swept across China and spread to many countries around the world. As of 25 March 2020, World Health Organization (WHO) reported 375498 cases and 16362 deaths related to COVID-19 worldwide.

COVID-19 mainly affects the respiratory tract, and the clinical manifestations are mostly fever, dry cough, fatigue, and dyspnea. In severe cases, it can develop into severe pneumonia, acute respiratory distress syndrome (ARDS), and multiple organ dysfunction (Huang et al., 2020). It is worth noting that an increasing number of cases show that in addition to the typical respiratory symptoms caused by 2019nCoV infection, it also causes cardiovascular complications, especially severe myocardial damage, which will aggravate the disease and impact the prognosis.

A recent study (Huang et al., 2020) reported that among the earliest 41 COVID-19 patients in Wuhan, five were diagnosed with acute myocardial injury $(12 \%)$, which was mainly manifested by elevated levels of high-sensitivity troponin I (hs-cTnl) $(>28 \mathrm{pg} / \mathrm{mL}$ ). In another recent report, of the 138 patients hospitalized for COVID-19, 16.7\% developed arrhythmia, and 7.2\% had acute myocardial injury (Wang et al., 2020). Among the earliest reported deaths in Wuhan, one patient was diagnosed with severe COVID-19associated myocarditis, with myocardial injury markers up to 20 times the upper limit of normal range and matched abnormal ECG manifestations.

In addition to direct myocardial damage, COVID-19 may also be a potential factor that induces instability of coronary plaques. Previous severe acute respiratory syndrome (SARS)-related clinical studies reported multiple cases complicated with acute myocardial infarction. Researchers believed that SARS coronavirus infection may cause dysregulation of inflammatory cytokines, such as elevated interleukin (IL) $-1 \beta$, interferon- $\gamma$, interferon-induced protein-10, and monocyte chemoattractant protein- 1 , which in turn trigger cytokine storms that cause myocardial damage and plaque instability, leading to myocardial infarction (Peiris et al., 2003; Tsui et al., 2003). Recent studies have shown that COVID-19 patients also have cytokine storms caused by the imbalance of Th1 and Th2 responses, and the levels of inflammatory factors IL -4 , IL-10 and IL-6 in tissue samples were increased (Huang et al., 2020; Chen et al., 2020).

According to the 4th edition of the internationally accepted definition of myocardial infarction (Thygesen et al., 2018), myocardial infarction is divided into 5 types, of which type 1 is plaque rupture or erosion of the coronary arteries, which in turn affects coronary blood flow and leads to myocardial infarction; type 2 defines the imbalance of myocardial blood supply / demand caused by specific factors leads to 
myocardial ischemic infarction. In our case, the angiography showed that the left anterior descending artery was severely stenotic along with thrombus formation, but the blood flow was TIMI grade 3. Based on the clinical characteristics of this case, we propose the possible mechanism of myocardial infarction: severe pulmonary infection, imbalance between coagulation and fibrinolysis subsequent to lung transplantation, changes in blood flow shear stress, and possible cytokine storms causing plaque rupture in the left anterior descending artery. In other words, both type 1 and type 2 myocardial infarction may participate in the patient's pathophysiological changes.

In terms of treating acute myocardial infarction, considering that the patient had recent 2019-nCoV infection and lung transplantation, we organized multiple rounds of multi-disciplinary discussions and decided that primary coronary intervention rather than thrombolytic therapy was suitable for the patient. Due to severe infection, recent lung transplantation, and a combination of high bleeding risks such as advanced age, declined platelet count, and prolonged prothrombin time, we adopted a tailored antithrombotic regime with dual antiplatelets of aspirin and clopidogrel for 3 days post-PCl, and single antiplatelet therapy with clopidogrel thereafter. We also intensified prevention of gastrointestinal bleeding with acid suppression by a proton pump inhibitor and gastric mucosa protection.

As the designated hospital for the treatment of critically ill patients with COVID-19 in Zhejiang province, our hospital has formulated detailed and complete plans for various situations. The designated cardiac catheterization lab is divided into a contaminated area, a semi-contaminated area, a buffer zone, and a clean area in advance. All the equipment and devices for the interventional procedures were prepared in place. In order to prevent cross contamination, all the items can only be transferred from the outside to the inside in one-way direction. Although our patient's nucleic acid test for 2019-nCoV had been negative for over 20 days, all the participating medical staff were equipped according to the standard three-level protection measures, including N95 masks, goggles, face protection masks and isolation gown, to reduce the potential risk of cross-infection. Coronary intervention was carried out in accordance with the expert consensus on cardiovascular management principles of COVID-19 recently released by the Chinese Society of Cardiology (CSC) (Han et al., 2020).

Finally, Although the patient was under ventilator-assisted ventilation and ECMO support, his lungs were progressively consolidated. Lung transplantation was considered after multidisciplinary discussion, and coronary computed tomography angiography (CTA) was recommended before the surgery. However, transporting this patient with so many supportive equipment was hardly possible and the patient's risk of going out for CT scan was extremely high. Thus, coronary CTA had not been performed before lung transplantation. This is also the experience and lessons we need to learn from this case.

\section{Conclusion}

This case is the first reported case of COVID-19 complicated with acute myocardial infarction treated with primary $\mathrm{PCl}$, and also the first case of acute myocardial infarction after bilateral lung transplantation. In addition to the patient's underlying severe coronary atherosclerosis, factors such as 
viral infection, inflammatory cytokine storm, hypoxia, blood loss, and bilateral lung transplantation may participate in the pathophysiology of myocardial infarction. With the interdisciplinary cooperation, we actively treat under the premise of adequate protection and decisively adopt the method of primary PCl to effectively stabilize the patient.

\section{Declarations}

\section{Contributors}

Tian-ming XUAN and Xiang-yuan PU analyzed the literatures and prepared the first draft of the manuscript. Xing-xiang WANG, Wei-li HAN and Xiao-gang GUO revised, edited, and checked the final version. All authors have read and approved the final manuscript.

\section{Compliance with ethics guidelines}

Tian-ming XUAN, Xing-xiang WANG, Xiang-yuan PU, Xiao-gang GUO and Wei-li HAN declare that they have no conflict of interest.

All procedures followed were in accordance with the ethical standards of the responsible committee on human experimentation (institutional and national) and with the Helsinki Declaration of 1975, as revised in 2008 (5). Informed consent was obtained from the patient for being included in the study.

\section{References}

1. Huang, C., Wang, Y., Li, X., et al., (2020). Clinical features of patients infected with 2019 novel coronavirus in Wuhan, China. Lancet, 395(10223), 497-506. https://doi.org/10.1016/S01406736(20)30183-5

2. Wang, D., Hu, B., Hu, C., et al., (2020). Clinical Characteristics of 138 Hospitalized Patients With 2019 Novel Coronavirus-Infected Pneumonia in Wuhan, China. JAMA, 323(11), 1061. https://doi.org/10.1001/jama.2020.1585

3. Website of Wuhan Municipal Health Commission, Hubei, China. http://wjw.wuhan.gov.cn/front/web/showDetail/2020011609057. Accessed Mar 26, 2020

4. Peiris, J., Chu, C., Cheng, V., et al., (2003). Clinical progression and viral load in a community outbreak of coronavirus-associated SARS pneumonia: a prospective study. Lancet, 361(9371), 1767-1772. https://doi.org/10.1016/S0140-6736(03)13412-5

5. Tsui, K.-L., Leung, T.-C., Yam, L. Y.-C., et al., (2005). Coronary plague instability in severe acute respiratory syndrome. Int J Cardiol, 99(3), 471-472. https://doi.org/10.1016/j.ijcard.2003.11.052 
6. Chen, N., Zhou, M., Dong, X., et al., (2020). Epidemiological and clinical characteristics of 99 cases of 2019 novel coronavirus pneumonia in Wuhan, China: a descriptive study. Lancet, 395(10223), 507513. https://doi.org/10.1016/S0140-6736(20)30211-7

7. Thygesen, K., Alpert, J. S., Jaffe, A. S., et al., (2018). Fourth Universal Definition of Myocardial Infarction (2018). J Am Coll Cardiol, 72(18), 2231-2264.

https://doi.org/10.1016/j.jacc.2018.08.1038

8. Han, Y., Zeng, H., Jiang, H., et al., (2020). CSC Expert Consensus on Principles of Clinical Management of Patients with Severe Emergent Cardiovascular Diseases during the COVID-19 Epidemic. Circulation, (83), CIRCULATIONAHA.120.047011. https://doi.org/10.1161/CIRCULATIONAHA.120.047011

\section{Figures}
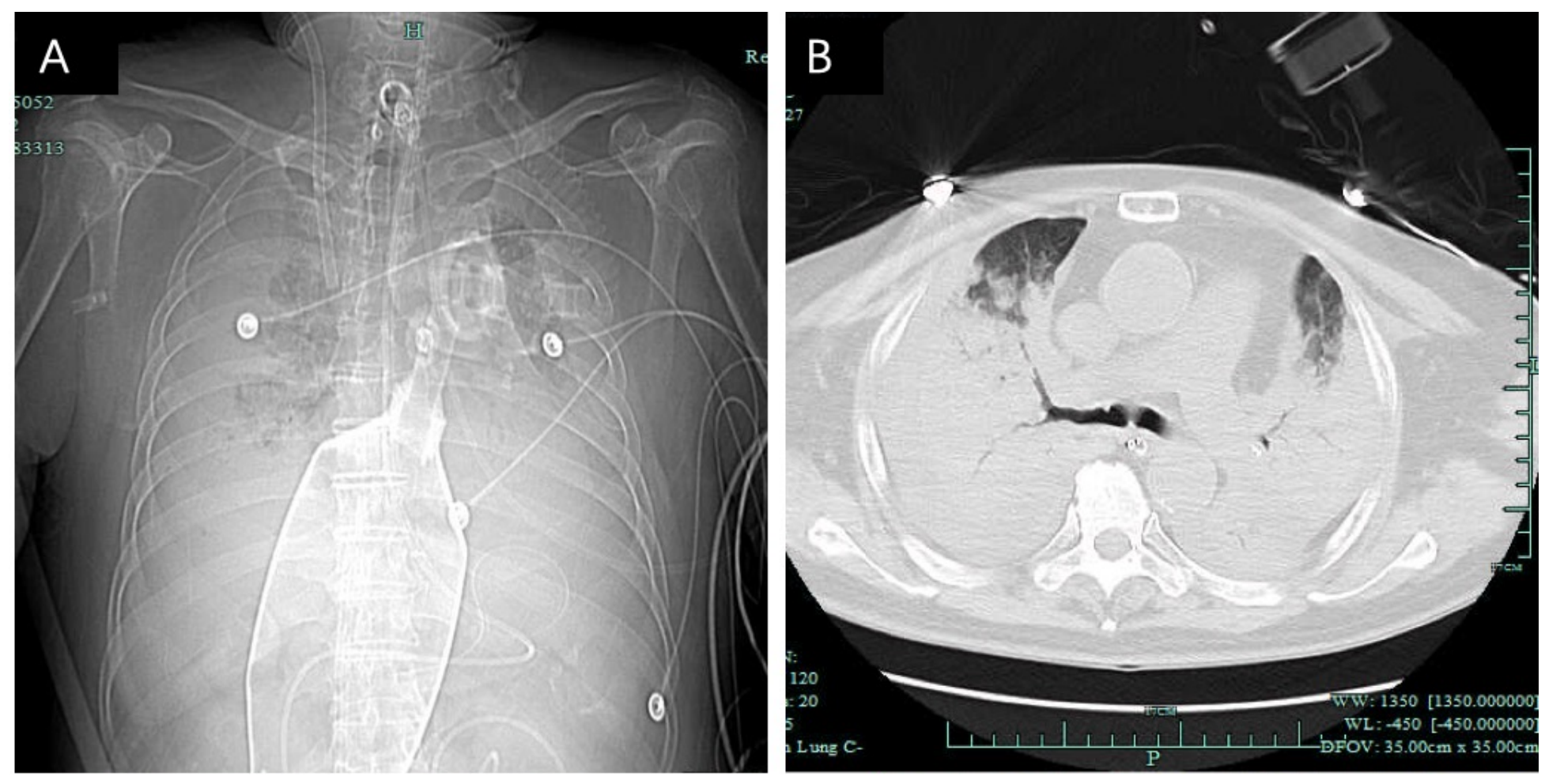

\section{Figure 1}

The chest X-ray (A) and CT (B) scan show severely diffused consolidation of the lung. 

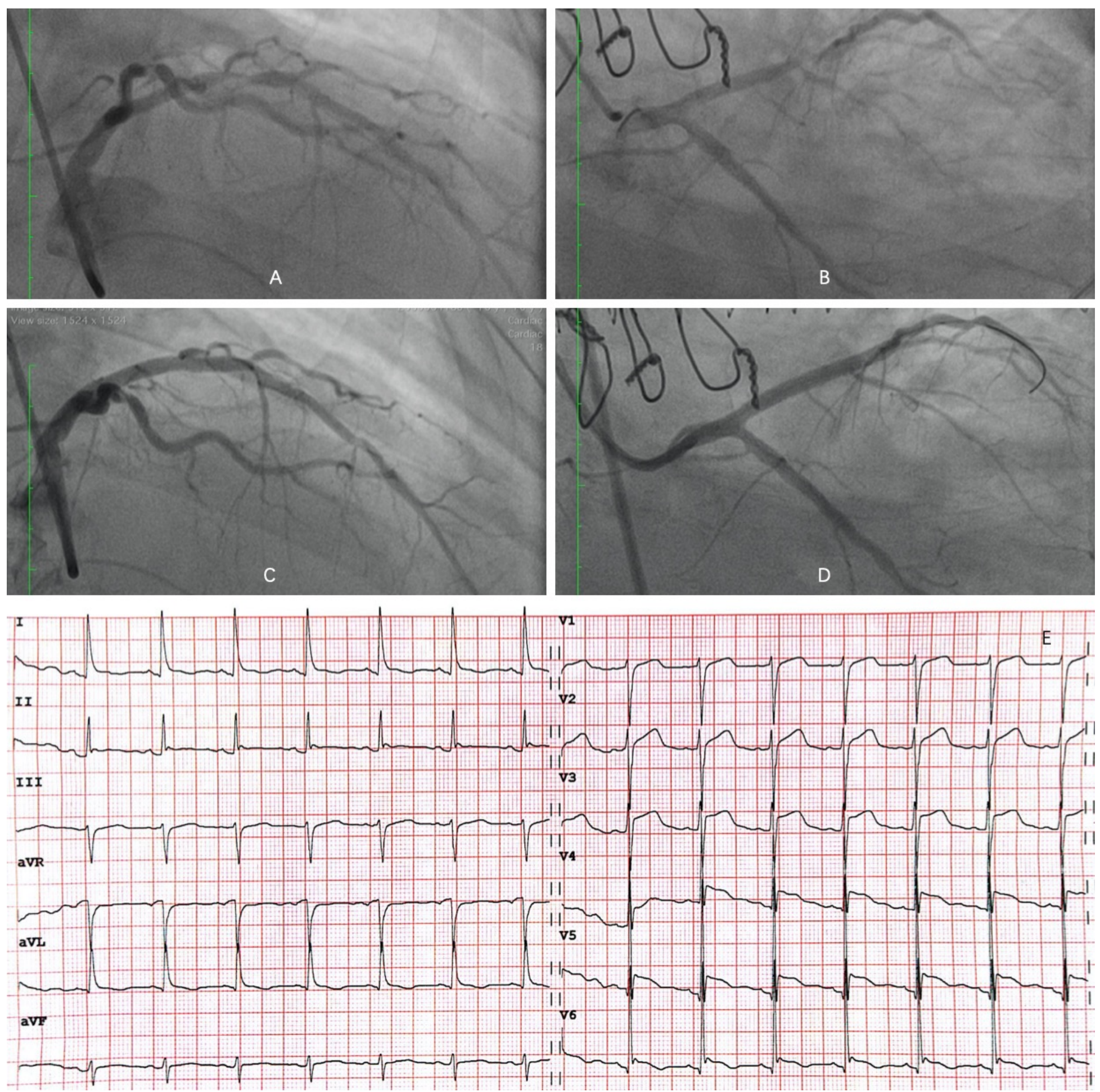

Figure 2

Angiogram before ( $A$ and $B$ ) and after ( $C$ and $D)$ primary percutaneous coronary intervention with stent implantation. (E) ECG shows diffuse ST-segment elevation through V1 to V6 leads. 